\title{
On the Complete Elliptic Integrals and Babylonian Identity II: An Approximation for the Complete Elliptic Integral of the first kind
}

\author{
Edigles Guedes ${ }^{1}$ and Prof. Dr. K. Raja Rama Gandhi ${ }^{2}$ \\ Number Theorist, Brazil ${ }^{1}$ \\ and \\ Resource person in Math for Oxford University Press and Professor in Math at BITS-Vizag ${ }^{2}$ \\ edigles.guedes@gmail.com
}

Abstract. Using the Theorem 4 of previous paper, I evaluate the complete elliptic integral of the first kind in approximate analytical closed form, by means of Bessel functions.

\section{Introduction}

By means of the theorem 4 of previous paper, I demonstrated the following approximations:

$$
K(k) \approx\left(\frac{\pi+\sqrt{\pi}}{2}\right)\left[I_{0}\left(\frac{k}{2}\right)\right]^{2}+\frac{\sqrt{\pi}}{2} k I_{0}\left(\frac{k}{2}\right) I_{1}\left(\frac{k}{2}\right)-\frac{\sqrt{\pi}\left(4+k^{2}\right)}{8}
$$

and

$$
K(k) \approx\left(\frac{\pi+\sqrt{\pi}}{2}\right)\left[I_{0}\left(\frac{k}{2}\right)\right]^{2}+\pi\left[I_{1}\left(\frac{k}{2}\right)\right]^{2}+\frac{7 \sqrt{\pi}}{8} k I_{0}\left(\frac{k}{2}\right) I_{1}\left(\frac{k}{2}\right)-\left(\frac{2 \pi k^{2}+7 \sqrt{\pi} k^{2}+16 \sqrt{\pi}}{32}\right),
$$

for $0<k \leq 1 / 2$

\section{THEOREMS}

Theorem 1. For $0<k \leq 1 / 2$, then

$$
K(k) \approx\left(\frac{\pi+\sqrt{\pi}}{2}\right)\left[I_{0}\left(\frac{k}{2}\right)\right]^{2}+\frac{\sqrt{\pi}}{2} k I_{0}\left(\frac{k}{2}\right) I_{1}\left(\frac{k}{2}\right)-\frac{\sqrt{\pi}\left(4+k^{2}\right)}{8},
$$

where $K(k)$ denotes the complete elliptic integral of first kind and $I_{v}(z)$ denotes the modified Bessel function of the first kind.

Proof. I will prove the equality above, developing the left hand side of the equation below

$$
K(k)-\frac{\pi}{2}\left[I_{0}\left(\frac{k}{2}\right)\right]^{2}=I_{k}
$$

First, I encounter

$$
\begin{gathered}
\frac{\pi}{2}\left[I_{0}\left(\frac{k}{2}\right)\right]^{2}=\frac{\sqrt{\pi}}{2} \sum_{m=0}^{\infty} \Gamma\left(m+\frac{1}{2}\right) \frac{k^{2 m}}{2^{2 m}(m !)^{3}}=\frac{\sqrt{\pi}}{2} \sum_{m=0}^{\infty} \frac{(2 m) !}{2^{2 m} m !} \cdot \frac{k^{2 m}}{2^{2 m}(m !)^{3}}=\frac{\sqrt{\pi}}{2} \sum_{m=0}^{\infty} \frac{(2 m) ! k^{2 m}}{2^{4 m}(m !)^{4}}(2) \\
=\frac{\sqrt{\pi}}{2} \sum_{m=0}^{\infty}\left(\begin{array}{c}
2 m \\
m
\end{array}\right) \frac{k^{2 m}}{2^{4 m}(m !)^{2}} .
\end{gathered}
$$

On the other hand, in [1], I proved the identity

$$
K(k)=\sqrt{\pi} \sum_{m=0}^{\infty} \frac{(2 m) ! \Gamma\left(m+\frac{3}{2}\right)}{(2 m+1)} \frac{k^{2 m}}{2^{2 m}(m !)^{3}}=\sqrt{\pi} \sum_{m=0}^{\infty} \frac{(2 m) !(2 m+2) !}{(2 m+1) 2^{2 m+2}(m+1) !} \frac{k^{2 m}}{2^{2 m}(m !)^{3}}
$$




$$
\begin{gathered}
=\sqrt{\pi} \sum_{m=0}^{\infty} \frac{(2 m) !(2 m+2)(2 m+1)(2 m) !}{(2 m+1) 2^{2 m+2}(m+1) m !} \cdot \frac{k^{2 m}}{2^{2 m}(m !)^{3}} \\
=\sqrt{\pi} \sum_{m=0}^{\infty} \frac{(2 m) ! 2(m+1)(2 m+1)(2 m) !}{(2 m+1) 2^{2 m+2}(m+1) m !} \cdot \frac{k^{2 m}}{2^{2 m}(m !)^{3}} \\
=\sqrt{\pi} \sum_{m=0}^{\infty} \frac{(2 m) !(2 m) !}{2^{2 m+1} m !} \cdot \frac{k^{2 m}}{2^{2 m}(m !)^{3}}=\frac{\sqrt{\pi}}{2} \sum_{m=0}^{\infty} \frac{(2 m) !}{2^{2 m} m !} \cdot \frac{(2 m) ! k^{2 m}}{2^{2 m}(m !)^{3}} \\
=\frac{\sqrt{\pi}}{2} \sum_{m=0}^{\infty} \frac{[(2 m) !]^{2} k^{2 m}}{2^{4 m}(m !)^{4}}=\frac{\sqrt{\pi}}{2} \sum_{m=0}^{\infty}\left(\begin{array}{c}
2 m \\
m
\end{array}\right)^{2} \frac{k^{2 m}}{2^{4 m}} .
\end{gathered}
$$

provided that $0<k<1$. I subtract (3) of (2), and obtain

$$
\begin{gathered}
K(k)-\frac{\pi}{2}\left[I_{0}\left(\frac{k}{2}\right)\right]^{2}=\frac{\sqrt{\pi}}{2} \sum_{m=0}^{\infty}\left(\begin{array}{c}
2 m \\
m
\end{array}\right)^{2} \frac{k^{2 m}}{2^{4 m}}-\frac{\sqrt{\pi}}{2} \sum_{m=0}^{\infty}\left(\begin{array}{c}
2 m \\
m
\end{array}\right) \frac{k^{2 m}}{2^{4 m}(m !)^{2}} \\
=\frac{\sqrt{\pi}}{2} \sum_{m=0}^{\infty}\left[\left(\begin{array}{c}
2 m \\
m
\end{array}\right)-\frac{1}{(m !)^{2}}\right]\left(\begin{array}{c}
2 m \\
m
\end{array}\right) \frac{k^{2 m}}{2^{4 m}} \\
=\frac{\sqrt{\pi}}{2} \sum_{m=0}^{\infty}\left[\frac{(2 m) !}{(m !)^{2}}-\frac{1}{(m !)^{2}}\right] \frac{(2 m) !}{(m !)^{2}} \frac{k^{2 m}}{2^{4 m}} \\
=\frac{\sqrt{\pi}}{2} \sum_{m=0}^{\infty}[(2 m) !-1] \frac{(2 m) !}{(m !)^{4}} \frac{k^{2 m}}{2^{4 m}} \\
=\frac{\sqrt{\pi}}{2} \sum_{m=1}^{\infty}[(2 m) !-1] \frac{(2 m) !}{(m !)^{4}} \frac{k^{2 m}}{2^{4 m}}=\frac{\sqrt{\pi} k^{2}}{2^{4}}+\frac{\sqrt{\pi}}{2} \sum_{m=2}^{\infty}[(2 m) !-1] \frac{(2 m) !}{(m !)^{4}} \frac{k^{2 m}}{2^{4 m}}
\end{gathered}
$$

I note that, if $m \geq 2$, then

$$
(2 m) !-1 \geq 5 m+1
$$

I take (5) into (4)

$$
\begin{gathered}
K(k)-\frac{\pi}{2}\left[I_{0}\left(\frac{k}{2}\right)\right]^{2}>\frac{\sqrt{\pi} k^{2}}{2^{4}}+\frac{\sqrt{\pi}}{2} \sum_{m=2}^{\infty}(5 m+1) \frac{(2 m) ! k^{2 m}}{(m !)^{4}} \frac{k^{4 m}}{2^{4 m}} \\
=\frac{\sqrt{\pi} k^{2}}{2^{4}}+\frac{5 \sqrt{\pi}}{2} \sum_{m=2}^{\infty} \frac{(2 m) ! m k^{2 m}}{(m !)^{4} 2^{4 m}}+\frac{\sqrt{\pi}}{2} \sum_{m=2}^{\infty} \frac{(2 m) ! k^{2 m}}{(m !)^{4} 2^{4 m}} \\
=\frac{\sqrt{\pi} k^{2}}{2^{4}}+\frac{5 \sqrt{\pi}}{2} \sum_{m=2}^{\infty}\left(\begin{array}{c}
2 m \\
m
\end{array}\right) \frac{k^{2 m}}{(m-1) ! m ! 2^{4 m}}+\frac{\sqrt{\pi}}{2} \sum_{m=2}^{\infty}\left(\begin{array}{c}
2 m \\
m
\end{array}\right) \frac{k^{2 m}}{(m !)^{2} 2^{4 m}} .
\end{gathered}
$$

I calculate

$\frac{5 \sqrt{\pi}}{2} \sum_{m=2}^{\infty}\left(\begin{array}{c}2 m \\ m\end{array}\right) \frac{k^{2 m}}{(m-1) ! m ! 2^{4 m}}=\frac{5 \sqrt{\pi} k^{2}}{16}{ }_{1} F_{2}\left(\frac{3}{2} ; 2,2 ; \frac{k^{2}}{4}\right)-\frac{5 \sqrt{\pi} k^{2}}{16}=\frac{5 \sqrt{\pi}}{4} k I_{0}\left(\frac{k}{2}\right) I_{1}\left(\frac{k}{2}\right)-\frac{5 \sqrt{\pi} k^{2}}{16}(7)$ 
and

$$
\frac{\sqrt{\pi}}{2} \sum_{m=2}^{\infty}\left(\begin{array}{c}
2 m \\
m
\end{array}\right) \frac{k^{2 m}}{(m !)^{2} 2^{4 m}}=\frac{\sqrt{\pi}}{2}{ }_{1} F_{2}\left(\frac{1}{2} ; 1,1 ; \frac{k^{2}}{4}\right)=-\frac{\sqrt{\pi} k^{2}}{16}+\frac{\sqrt{\pi}}{2}\left[I_{0}\left(\frac{k}{2}\right)\right]^{2}-\frac{\sqrt{\pi}}{2} .
$$

From (6), (7) and (8), I obtain hence,

$$
K(k)-\frac{\pi}{2}\left[I_{0}\left(\frac{k}{2}\right)\right]^{2}>\frac{\sqrt{\pi} k^{2}}{16}+\frac{5 \sqrt{\pi}}{4} k I_{0}\left(\frac{k}{2}\right) I_{1}\left(\frac{k}{2}\right)-\frac{5 \sqrt{\pi} k^{2}}{16}-\frac{\sqrt{\pi} k^{2}}{16}+\frac{\sqrt{\pi}}{2}\left[I_{0}\left(\frac{k}{2}\right)\right]^{2}-\frac{\sqrt{\pi}}{2},
$$

$$
K(k)>\left(\frac{\pi+\sqrt{\pi}}{2}\right)\left[I_{0}\left(\frac{k}{2}\right)\right]^{2}+\frac{5 \sqrt{\pi}}{4} k I_{0}\left(\frac{k}{2}\right) I_{1}\left(\frac{k}{2}\right)-\sqrt{\pi}\left(\frac{5 k^{2}+8}{16}\right),
$$

since $0<k<1 / 2$, I conclude easily that

$$
K(k) \approx\left(\frac{\pi+\sqrt{\pi}}{2}\right)\left[I_{0}\left(\frac{k}{2}\right)\right]^{2}+\frac{5 \sqrt{\pi}}{4} k I_{0}\left(\frac{k}{2}\right) I_{1}\left(\frac{k}{2}\right)-\sqrt{\pi}\left(\frac{5 k^{2}+8}{16}\right) .
$$

This completed the proof. The reader can see the Table 1.

Theorem 2. For $0<k \leq 1 / 2$, then

$$
K(k) \approx\left(\frac{\pi+\sqrt{\pi}}{2}\right)\left[I_{0}\left(\frac{k}{2}\right)\right]^{2}+\pi\left[I_{1}\left(\frac{k}{2}\right)\right]^{2}+\frac{7 \sqrt{\pi}}{8} k I_{0}\left(\frac{k}{2}\right) I_{1}\left(\frac{k}{2}\right)-\left(\frac{2 \pi k^{2}+7 \sqrt{\pi} k^{2}+16 \sqrt{\pi}}{32}\right),
$$

where $K(k)$ denotes the complete elliptic integral of first kind and $I_{v}(z)$ denotes the modified Bessel function of the first kind.

Proof. I will prove the equality above, developing the left hand side of the equation below

$$
K(k)-\left\{\frac{\pi}{2}\left[I_{0}\left(\frac{k}{2}\right)\right]^{2}+\pi\left[I_{1}\left(\frac{k}{2}\right)\right]^{2}\right\}=I_{k}
$$

First, I encounter

$$
\begin{gathered}
\frac{\pi}{2}\left[I_{0}\left(\frac{k}{2}\right)\right]^{2}=\frac{\sqrt{\pi}}{2} \sum_{m=0}^{\infty} \Gamma\left(m+\frac{1}{2}\right) \frac{k^{2 m}}{2^{2 m}(m !)^{3}}=\frac{\sqrt{\pi}}{2} \sum_{m=0}^{\infty} \frac{(2 m) !}{2^{2 m} m !} \cdot \frac{k^{2 m}}{2^{2 m}(m !)^{3}}=\frac{\sqrt{\pi}}{2} \sum_{m=0}^{\infty} \frac{(2 m) ! k^{2 m}}{2^{4 m}(m !)^{4}} \\
=\frac{\sqrt{\pi}}{2} \sum_{m=0}^{\infty}\left(\begin{array}{c}
2 m \\
m
\end{array}\right) \frac{k^{2 m}}{2^{4 m}(m !)^{2}}
\end{gathered}
$$

And

$$
\begin{aligned}
& \pi\left[I_{1}\left(\frac{k}{2}\right)\right]^{2}=\sqrt{\pi} \sum_{m=0}^{\infty} \frac{\Gamma\left(\frac{2 m+1}{2}\right)}{\Gamma(m+1) \Gamma(m+2) \Gamma(m)} \frac{k^{2 m}}{2^{2 m}}=\sqrt{\pi} \sum_{m=0}^{\infty} \frac{\Gamma\left(m+\frac{1}{2}\right)}{(m-1) ! m !(m+1) !} \frac{k^{2 m}}{2^{2 m}} \\
& =\pi \sum_{m=0}^{\infty} \frac{(2 m) !}{(m-1) !(m !)^{2}(m+1) !} \frac{k^{2 m}}{2^{4 m}}=\pi \sum_{m=0}^{\infty}\left(\begin{array}{c}
2 m \\
m
\end{array}\right) \frac{k^{2 m}}{(m-1) !(m+1) ! 2^{4 m}}
\end{aligned}
$$


On the other hand, in [1], I proved the identity

$$
\begin{gathered}
K(k)=\sqrt{\pi} \sum_{m=0}^{\infty} \frac{(2 m) ! \Gamma\left(m+\frac{3}{2}\right)}{(2 m+1)} \frac{k^{2 m}}{2^{2 m}(m !)^{3}}=\sqrt{\pi} \sum_{m=0}^{\infty} \frac{(2 m) !(2 m+2) !}{(2 m+1) 2^{2 m+2}(m+1) !} \frac{k^{2 m}}{2^{2 m}(m !)^{3}} \\
=\sqrt{\pi} \sum_{m=0}^{\infty} \frac{(2 m) !(2 m+2)(2 m+1)(2 m) !}{(2 m+1) 2^{2 m+2}(m+1) m !} \cdot \frac{k^{2 m}}{2^{2 m}(m !)^{3}} \\
=\sqrt{\pi} \sum_{m=0}^{\infty} \frac{(2 m) ! 2(m+1)(2 m+1)(2 m) !}{(2 m+1) 2^{2 m+2}(m+1) m !} \cdot \frac{k^{2 m}}{2^{2 m}(m !)^{3}} \\
=\sqrt{\pi} \sum_{m=0}^{\infty} \frac{(2 m) !(2 m) !}{2^{2 m+1} m !} \cdot \frac{k^{2 m}}{2^{2 m}(m !)^{3}}=\frac{\sqrt{\pi}}{2} \sum_{m=0}^{\infty} \frac{(2 m) !}{2^{2 m} m !} \cdot \frac{(2 m) ! k^{2 m}}{2^{2 m}(m !)^{3}} \\
=\frac{\sqrt{\pi}}{2} \sum_{m=0}^{\infty} \frac{[(2 m) !]^{2} k^{2 m}}{2^{4 m}(m !)^{4}}=\frac{\sqrt{\pi}}{2} \sum_{m=0}^{\infty}\left(\begin{array}{c}
2 m \\
m
\end{array}\right)^{2} \frac{k^{2 m}}{2^{4 m}}
\end{gathered}
$$

provided that $0<k<1$. I subtract (12) of (11) and (10), to obtain

$$
\begin{aligned}
K(k)-\left\{\frac{\pi}{2}\left[I_{0}\left(\frac{k}{2}\right)\right]^{2}+\pi\left[I_{1}\left(\frac{k}{2}\right)\right]^{2}\right\} \\
=\frac{\sqrt{\pi}}{2} \sum_{m=0}^{\infty}\left(\begin{array}{c}
2 m \\
m
\end{array}\right)^{2} \frac{k^{2 m}}{2^{4 m}}-\frac{\sqrt{\pi}}{2} \sum_{m=0}^{\infty}\left(\begin{array}{c}
2 m \\
m
\end{array}\right) \frac{k^{2 m}}{2^{4 m}(m !)^{2}}-\pi \sum_{m=0}^{\infty}\left(\begin{array}{c}
2 m \\
m
\end{array}\right) \frac{k^{2 m}}{(m-1) !(m+1) ! 2^{4 m}} \\
=\sqrt{\pi} \sum_{m=0}^{\infty}\left[\frac{1}{2}\left(\begin{array}{c}
2 m \\
m
\end{array}\right)-\frac{1}{2(m !)^{2}}-\frac{\sqrt{\pi}}{(m-1) !(m+1) !}\right]\left(\begin{array}{c}
2 m \\
m
\end{array}\right) \frac{k^{2 m}}{2^{4 m}} \\
=\sqrt{\pi} \sum_{m=0}^{\infty}\left[\frac{1}{2} \frac{(2 m) !}{(m !)^{2}}-\frac{1}{2(m !)^{2}}-\frac{\sqrt{\pi}}{(m-1) !(m+1) !}\right] \frac{(2 m) !}{(m !)^{2}} \frac{k^{2 m}}{2^{4 m}} \\
=\frac{\sqrt{\pi}}{2} \sum_{m=0}^{\infty}\left[\frac{(2 m) !}{(m !)^{2}}-\frac{1}{(m !)^{2}}-\frac{2 m \sqrt{\pi}}{m(m-1) !(m+1) m !}\right] \frac{(2 m) !}{(m !)^{2}} \frac{k^{2 m}}{2^{4 m}} \\
=\frac{\sqrt{\pi}}{2} \sum_{m=0}^{\infty}\left[\frac{(2 m) !}{(m !)^{2}}-\frac{1}{(m !)^{2}}-\frac{2 m \sqrt{\pi}}{(m !)^{2}(m+1)}\right] \frac{(2 m) !}{(m !)^{2}} \frac{k^{2 m}}{2^{4 m}} \\
=\frac{\sqrt{\pi}}{2} \sum_{m=0}^{\infty}\left[(2 m) !-1-\frac{2 m \sqrt{\pi}}{m+1}\right] \frac{(2 m) !}{(m !)^{4}} \frac{k^{2 m}}{2^{4 m}} \\
=\frac{\sqrt{\pi}}{2} \sum_{m=1}^{\infty}\left[(2 m) !-1-\frac{2 m \sqrt{\pi}}{m+1}\right] \frac{(2 m) !}{(m !)^{4}} \frac{k^{2 m}}{2^{4 m}} \\
=\frac{\sqrt{\pi}}{2} \sum_{m=2}^{\infty}\left[(2 m) !-1-\frac{2 m \sqrt{\pi}}{m+1}\right] \frac{(2 m) !}{(m !)^{4}} \frac{k^{2 m}}{2^{4 m}} \\
=
\end{aligned}
$$


I note that, if $m \geq 2$, then

$$
(2 m) !-1-\frac{2 m \sqrt{\pi}}{m+1}>\frac{7 m}{2}+1
$$

I take (14) into (13)

$$
\begin{aligned}
& K(k)-\left\{\frac{\pi}{2}\left[I_{0}\left(\frac{k}{2}\right)\right]^{2}+\pi\left[I_{1}\left(\frac{k}{2}\right)\right]^{2}\right\}>\frac{\sqrt{\pi}}{2} \sum_{m=2}^{\infty}\left(\frac{7 m}{2}+1\right) \frac{(2 m) !}{(m !)^{4}} \frac{k^{2 m}}{2^{4 m}}-\left(\frac{\pi-\sqrt{\pi}}{16}\right) k^{2} \\
& =\frac{7 \sqrt{\pi}}{4} \sum_{m=2}^{\infty} \frac{(2 m) ! m k^{2 m}}{(m !)^{4} 2^{4 m}}+\frac{\sqrt{\pi}}{2} \sum_{m=2}^{\infty} \frac{(2 m) ! k^{2 m}}{(m !)^{4} 2^{4 m}}-\left(\frac{\pi-\sqrt{\pi}}{16}\right) k^{2} \\
& =\frac{7 \sqrt{\pi}}{4} \sum_{m=2}^{\infty}\left(\begin{array}{c}
2 m \\
m
\end{array}\right) \frac{k^{2 m}}{(m-1) ! m ! 2^{4 m}}+\frac{\sqrt{\pi}}{2} \sum_{m=2}^{\infty}\left(\begin{array}{c}
2 m \\
m
\end{array}\right) \frac{k^{2 m}}{(m !)^{2} 2^{4 m}}-\left(\frac{\pi-\sqrt{\pi}}{16}\right) k^{2} .
\end{aligned}
$$

I calculate

$$
\frac{7 \sqrt{\pi}}{4} \sum_{m=2}^{\infty}\left(\begin{array}{c}
2 m \\
m
\end{array}\right) \frac{k^{2 m}}{(m-1) ! m ! 2^{4 m}}=\frac{7 \sqrt{\pi} k^{2}}{32}{ }_{1} F_{2}\left(\frac{3}{2} ; 2,2 ; \frac{k^{2}}{4}\right)-\frac{7 \sqrt{\pi} k^{2}}{32}=\frac{7 \sqrt{\pi}}{8} k I_{0}\left(\frac{k}{2}\right) I_{1}\left(\frac{k}{2}\right)-\frac{7 \sqrt{\pi} k^{2}}{32}
$$

and

$$
\frac{\sqrt{\pi}}{2} \sum_{m=2}^{\infty}\left(\begin{array}{c}
2 m \\
m
\end{array}\right) \frac{k^{2 m}}{(m !)^{2} 2^{4 m}}=\frac{\sqrt{\pi}}{2}{ }_{1} F_{2}\left(\frac{1}{2} ; 1,1 ; \frac{k^{2}}{4}\right)=-\frac{\sqrt{\pi} k^{2}}{16}+\frac{\sqrt{\pi}}{2}\left[I_{0}\left(\frac{k}{2}\right)\right]^{2}-\frac{\sqrt{\pi}}{2} .
$$

From (15), (16) and (17), I obtain

$$
K(k)>\left(\frac{\pi+\sqrt{\pi}}{2}\right)\left[I_{0}\left(\frac{k}{2}\right)\right]^{2}+\pi\left[I_{1}\left(\frac{k}{2}\right)\right]^{2}+\frac{7 \sqrt{\pi}}{8} k I_{0}\left(\frac{k}{2}\right) I_{1}\left(\frac{k}{2}\right)-\left(\frac{2 \pi k^{2}+7 \sqrt{\pi} k^{2}+16 \sqrt{\pi}}{32}\right),
$$

since $0<k<1 / 2$, I conclude easily that

$$
K(k) \approx\left(\frac{\pi+\sqrt{\pi}}{2}\right)\left[I_{0}\left(\frac{k}{2}\right)\right]^{2}+\pi\left[I_{1}\left(\frac{k}{2}\right)\right]^{2}+\frac{7 \sqrt{\pi}}{8} k I_{0}\left(\frac{k}{2}\right) I_{1}\left(\frac{k}{2}\right)-\left(\frac{2 \pi k^{2}+7 \sqrt{\pi} k^{2}+16 \sqrt{\pi}}{32}\right) .
$$

This completed the proof. The reader can see the Table 2 . 
Table 1

In this table, I have: first column: $m$; second column: $k=1 / m$; third column: $\left(\frac{\pi+\sqrt{\pi}}{2}\right)\left[I_{0}\left(\frac{1}{2 m}\right)\right]^{2}+\frac{5 \sqrt{\pi}}{4 m} I_{0}\left(\frac{1}{2 m}\right) I_{1}\left(\frac{1}{2 m}\right)-\frac{\sqrt{\pi}}{16}\left[5\left(\frac{1}{m}\right)^{2}+8\right]$; fourth column: $K\left(\frac{1}{m}\right)$; fifth column: $\frac{K\left(\frac{1}{m}\right)}{\left(\frac{\pi+\sqrt{\pi}}{2}\right)\left[I_{0}\left(\frac{1}{2 m}\right)\right]^{2}+\frac{5 \sqrt{\pi}}{4 m} I_{0}\left(\frac{1}{2 m}\right) I_{1}\left(\frac{1}{2 m}\right)-\frac{\sqrt{\pi}}{16}\left[5\left(\frac{1}{m}\right)^{2}+8\right]}$.

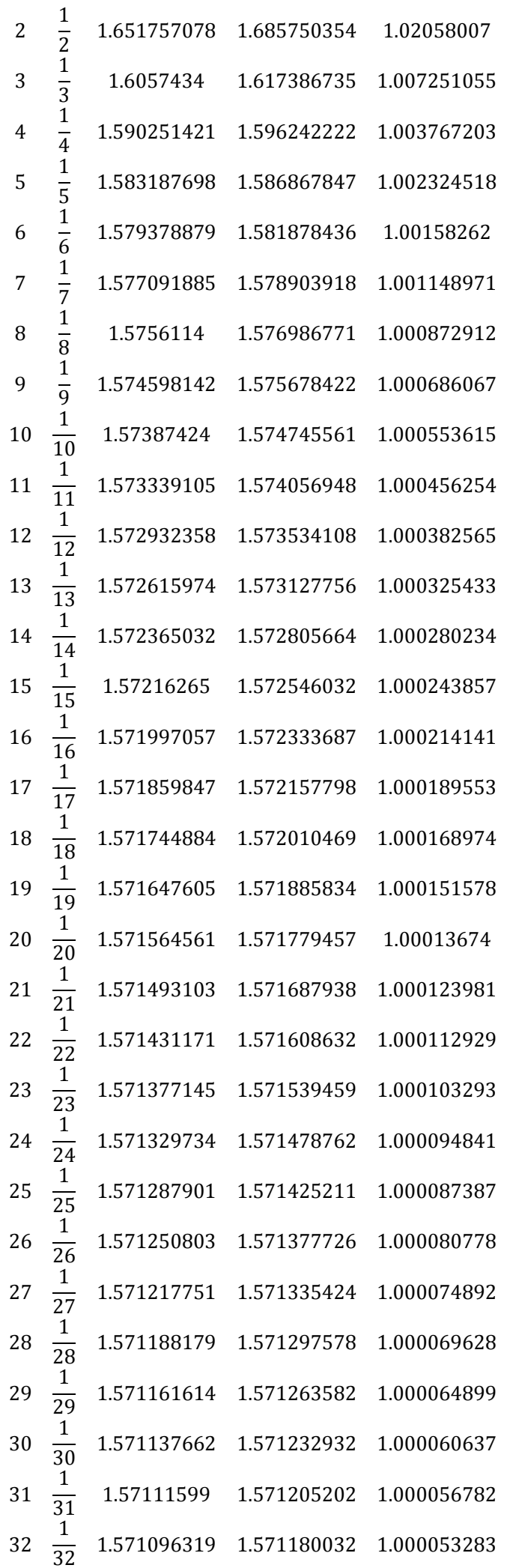


Table 2

In this table, I have: first column: $m$; second column: $k=1 / m$; third column: $\left(\frac{\pi+\sqrt{\pi}}{2}\right)\left[I_{0}\left(\frac{1}{2 m}\right)\right]^{2}+\pi\left[I_{1}\left(\frac{1}{2 m}\right)\right]^{2}+\frac{7 \sqrt{\pi}}{8 m} I_{0}\left(\frac{1}{2 m}\right) I_{1}\left(\frac{1}{2 m}\right)-\left(\frac{2 \pi(1 / m)^{2}+7 \sqrt{\pi}(1 / m)^{2}+16 \sqrt{\pi}}{32}\right) ; \quad$ fourth column: $K\left(\frac{1}{m}\right)$; fifth column: $\frac{K\left(\frac{1}{m}\right)}{\left(\frac{\pi+\sqrt{\pi}}{2}\right)\left[I_{0}\left(\frac{1}{2 m}\right)\right]^{2}+\pi\left[I_{1}\left(\frac{1}{2 m}\right)\right]^{2}+\frac{7 \sqrt{\pi}}{8 m} I_{0}\left(\frac{1}{2 m}\right) I_{1}\left(\frac{1}{2 m}\right)-\left(\frac{2 \pi(1 / m)^{2}+7 \sqrt{\pi}(1 / m)^{2}+16 \sqrt{\pi}}{32}\right)}$.

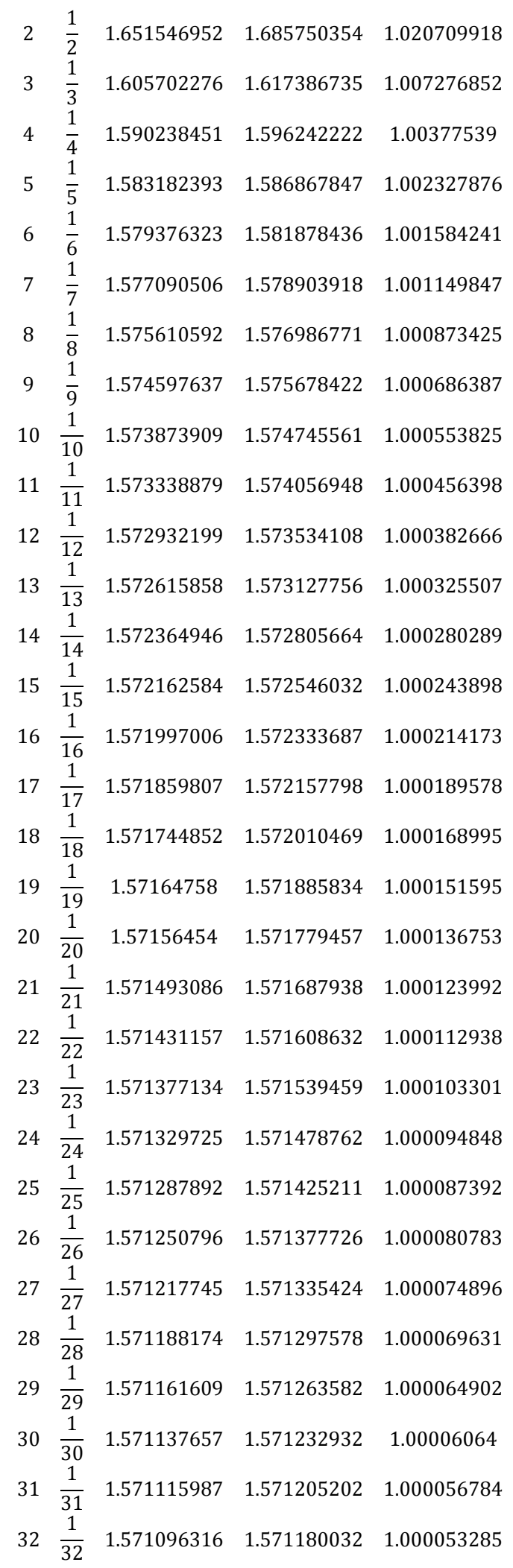




\section{REFERENCES}

[1] Guedes, Edigles, On the Complete Elliptic Integrals and Babylonian Identity I: The 1/ $\pi$ formulaes Involving Gamma functions and Summations, 2013. 